\title{
A generic method to develop simulation models for ambulance systems
}

\section{R. Pinto ${ }^{a, 1}$, P. M. S. Silva ${ }^{a}$, T. P. Young ${ }^{b}$}

a Universidade Federal de Minas Gerais, Av. Presidente Antônio Carlos, 6627, Belo Horizonte, 31270-901, MG, Brazil

b Brunel University London, Kingston Lane, Uxbridge, Middlesex, UB8 3PH, UK

\section{Abstract}

In this paper, we address the question of generic simulation models and their role in improving emergency care around the world. After reviewing the development of ambulance models and the contexts in which they have been applied, we report the construction of a reusable model for ambulance systems. Further, we describe the associated parameters, data sources, and performance measures, and report on the collection of information, as well as the use of optimisation to configure the service to best effect. Having developed the model, we have validated it using real data from the emergency medical system in a Brazilian city, Belo Horizonte. To illustrate the benefits of standardisation and reusability we apply the model to a UK context by exploring how different rules of engagement would change the performance of the system. Finally, we consider the impact that one might observe if such rules were adopted by the Brazilian system.

Keywords: simulation, modelling, healthcare, ambulance services.

\section{Introduction}

The reuse of models is a topic of considerable interest in healthcare because, although most care delivery systems are specifically designed for the local context, many share common design elements. As an example, emergency departments share common operational principles but the configuration, management and layout of one department will differ from another. In this paper, we use an example from emergency medicine - ambulance control - as an exemplar with which to explore the question of reuse and standardisation of models.

Ambulance services are attractive to model for a number of reasons. First, the start and end of each task is well defined, tasks are self-contained and take place over

\footnotetext{
${ }^{1}$ Corresponding author. Tel. +55 3134094901

E-mail address: luiz@dep.ufmg.br (L. R. Pinto)

This paper has been published at Simulation Modelling Practice and Theory 51 (2015) 170-183

DOI: http://dx.doi.org/10.1016/i.simpat.2014.12.001. Please use this link to cite the paper.
} 
short periods of time. Moreover, the processes lend themselves to a logistic analysis well-suited to modelling. This is not the case for all medical services, many of which are open ended, run concurrently with other medical interventions and stretch over long periods of time. Second, as we shall see, although there are many dimensions of uncertainty to modelling an ambulance service, these dimensions can be partitioned with relative ease and changed when moving from one ambulance service to another.

The ability to expand a model by interfacing it to another, even off-the-shelf, model is a third feature of reuse and standardisation. The geographical spread of ambulance services provides an opportunity to explore geographical information systems (GIS) and thus to extend a model's applicability from one city to another. Fourth, the high number of variables and the random nature of demand make the analysis for decision-making a combinatorial problem with a high number of alternatives and renders deterministic methods unattractive.

Finally, setting appropriate metrics is a case of assessing complex trade-offs, rather than applying measures that have been derived from first principles. For instance, an ambulance in front of every house would minimise the response time (the elapsed time from the call to the arrival of an ambulance on the scene), but at prohibitive cost. To satisfy such demand with a high quality service, it is required a redundant system, with a low utilisation of resource in consequence. Meanwhile, system managers are pressed to operate the system within cost constraints, which implies maximising the utilisation of resource.

For all these reasons, simulation modelling is an attractive way of analysing such scenarios. Section 2 surveys the vast literature on modelling and simulation that has been applied to ambulance services. However, most Discrete Event Simulation (DES) models are built for a specific city in order to analyse a specific aspect of the system, making reuse hard if not impossible.

In this paper, we propose a generic DES modelling for ambulance services. The proposed modelling is comprehensive and emphasises the use of modular approach in order to build generic models. The methodology describes in detail, all stages we usually take during a simulation analysis of an Emergency Medical System (EMS). As a basis, we have taken a specific model [1,2] and generalised it to capture features of other systems. Section 3 describes ambulance services in generic terms, the overall structure of the model and the associated parameters, the data sources and collection, and the construction of an optimising loop around the simulation. In 
section 4 we explain the validation of the model and the results are discussed in section 5 , with a short conclusion to clarify the findings (section 6).

\section{Literature review}

Ambulance services have been analysed in a variety of ways, including queuing theory and hypercube models $[3,4,5,6,7,8,9]$, mixed integer programming [10, 11], stochastic optimization [12] and dynamic programming [13]. By contrast, simulation modelling presents a high cost of implementation, but gives a more detailed description of the system [3] and allows the analysis of dynamic effects [14, 15]. These features make the simulation approach a powerful decision-making tool for analysing such systems.

Simulation models have been used to analyse ambulance services since the ' $60 \mathrm{~s}$ $[16,17,18,19,20]$. Since then, there have been many studies focused on a particular city or region.

Fujiwara et al. [21] analysed deployment policies for Bangkok, Thailand, combining a simulation model informed by a location model (a mathematical programming model). Meanwhile, Henderson and Mason [22] built a simulation model to refine the solutions proposed by a queue model for the ambulance service of Auckland, New Zealand.

Kock and Weigl [23] used simulation to analyse ambulance logistics in Austria, with the main objective of comparing centralised and decentralised policies of transportation for the Austrian Red Cross. Su and Shih [24] developed a simulation model of the ambulance service system of Taipei, Taiwan and used it to explore alternative scenarios that might improve the operation of the system.

Wu and Hwang [25] built a wide-ranging simulation model to analyse the ambulance system of Tainan, Taiwan. This model was used to estimate the threshold for expanding the ambulance fleet and to evaluate ambulance-dispatching strategies during large public events such as concerts and marathon races.

Silva and Pinto [2] developed a model to analyse the ambulance service of Belo Horizonte, Brazil. The model was used to analyse two aspects of the service: its response to increased demand and what expansion of the fleet would be needed to reduce the average response time significantly.

Meanwhile, Berchi et al. [14] proposed a five-step methodology to planning the ambulance service system of Milan, Italy. The proposed method was used to estimate the preferred locations of ambulances depots and number of ambulances. 
More recently, Aboueljinane et al. [26] developed a simulation study to improve the system of Val-de-Marne department, a small administrative district located in the southeast of Paris, France. Zhen et al. [27] proposed a simulation optimisation method in order to evaluate the performance of ambulance deployment and relocations plans. The authors run a demo example using the system of Shanghai, China. Morohosi and Furuta [9] built and applied a simulation model to a large-scale ambulance system in the Tokyo metropolis.

It will be noted that many of these exercises involved another mathematical technique in conjunction with the simulation model. Morohosi and Furuta [15] applied the MEXCLP (maximum expected covering location problem) location model to ambulance data in Tokyo metropolis and they verified the assumptions of the model via simulation. Aringhieri [28] took this further by developing a hybrid approach that integrated Agent Based Simulation (ABS) and Discrete Event Simulation (DES) to analyse the ambulance service system, again in Milan. The author states that the model is quite flexible and could be used in other cities of Lombardy. Zhen et al. [27] proposed a simulation-optimisation method in order to evaluate the performance of ambulance deployment plans. The authors used a genetic algorithm to guide the search process. The key point is that simulation within a geographical context has been routinely demonstrated, but geographic reusability remains an elusive, but attractive goal.

More broadly, the application of simulation modelling and other OR techniques to healthcare has been widely discussed in the literature. This is driven by such factors as the increasing and aging population, especially in urban centres $[29,30]$ with a knock-on effect for ambulance services. Comparisons with manufacturing and military usage have revealed a significant lack impact for healthcare modelling [31, 32], and there has been a discussion of the barriers faced in healthcare [33, 34]. Notwithstanding, the number of simulation and modelling papers in healthcare has increased since than 2004 [35]. The pressure for better services, the low availability of resources and the need to assess the impact of changes before its actual implementation can be seen as an opportunity to increase Modelling and Simulation (M\&S) in healthcare [36].

Young et al. [34] have suggested the use of generic models as a strategy to improve the adoption of $O R$ techniques in healthcare, enabling service managers to set up models or reuse them in analysing their specific systems. This work is consonant with earlier findings. Fone et al. [37] presented an extended literature 
review of OR applications in healthcare and concluded that the quality of models had improved from 1980 until 1999 but noted that most models analyse the micro level of the system: specific aspects of some part of healthcare systems, such as Accident and Emergency (A\&E) or the departments of a hospital. They point to the lack of more generic models with wider applicability.

In the same vein, Gunal and Pidd [35] concluded that despite the increasing number of publications in healthcare since 2004 there is still a lack of generic methodologies to analyse healthcare systems. They argue that the models are very specific for each case and the reuse of the models is difficult. Again, we are able to draw a general conclusion that generic models are an elusive aspiration of the wider simulation and modelling community in healthcare.

In terms of optimisation, amongst the cited papers only Silva and Pinto [2] and Zhen et al. [27] have optimised the model's performance in integrated way [38]. In all other cases, when simulation and optimisation models were used together, the models are run separately using the following general algorithm. First, the model is run with any stochastic parameter replaced by an average value. Second, the simulation model is run to evaluate the proposed solutions in a dynamic and more realistic context, where the stochastic parameters are represented by probabilistic distribution. On such a basis, the optimisation models are used as an initial approximation and their solutions should be checked and/or refined in a simulation model.

This paper proposes a generic method to develop simulation models of ambulance service systems. Models found in literature are usually built for a specific system and could not be reused without great relative effort.

The method is comprehensive and it proposes the following guidelines: $(i)$ analysis of the main input parameters, (ii) development of the simulation model, (iii) analysis of the performance measures, and (iv) use of optimisation for simulation to analyse scenarios.

This method can improve the development of decision-making tools in order to analyse ambulance systems. Furthermore, we will be able to build models in an easy and quick way and the models can be tailored and then reused. In order to ease the input setup of the model, the method proposes a standardization of input parameters. We give an example of reusability in a practical way, applying some policies used for an EMS of UK in an EMS of Brazil in order to investigate likely improvements.

We believe that this method takes many steps towards filling the gaps that still exist in modelling and simulation (M\&S) of ambulance service systems. 


\section{The ambulance model}

A generic model to illustrate how ambulance systems work is shown in Fig. 1. The model represented by solid and dotted lines is the static dispatch model where ambulances are only dispatched from bases. On the other hand, the model represented by a combination of solid and dashed lines is a multi-location dispatch model, where the ambulances may be dispatched from wherever they are. Another approach, which is not represented here, is the dynamic dispatch, where the ambulance may be rerouted at any time in order to serve a higher priority call. In this case the system needs to know the position of the ambulance at all times, in order to effect an appropriate dispatch, i.e., the system should use a Geographical Information System - GIS.

The system has three main cores: (i) call generation (A), (ii) dispatch of ambulances (B), and (iii) ambulance journey (C). Although the three cores have complex processes, they follow similar rules for all systems, and we will provide some directions to build the simulation model.

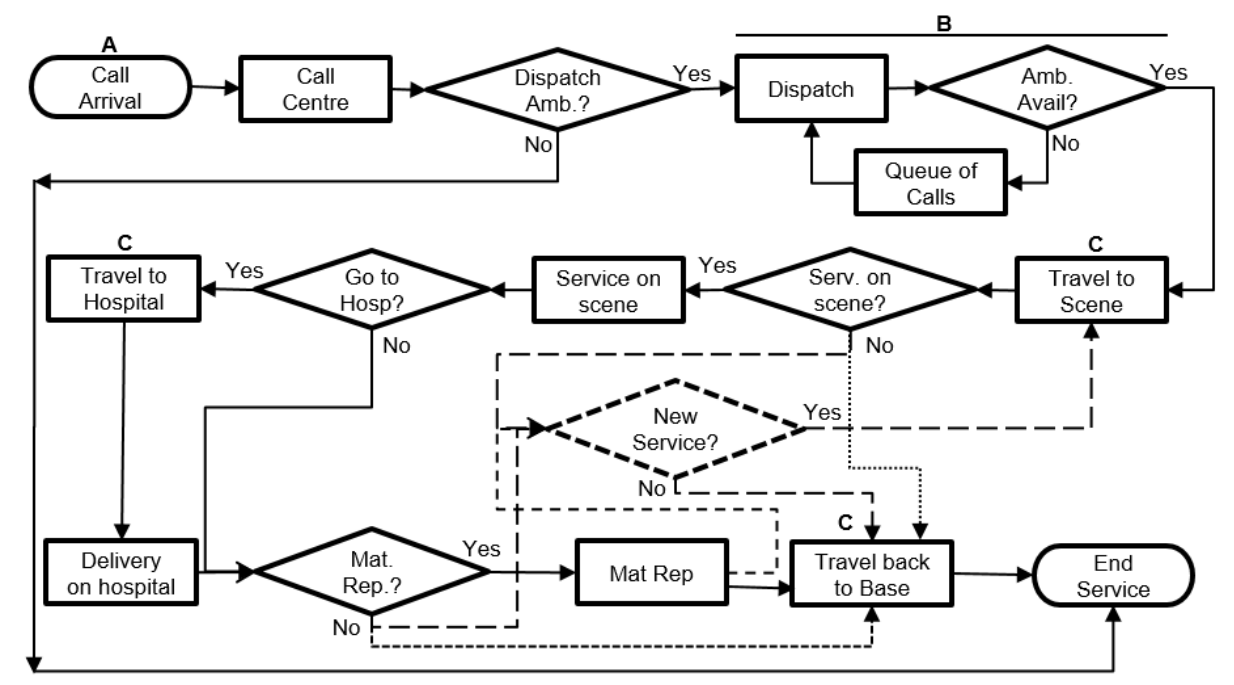

Figure 1: Model of ambulance system

The overall structure of the simulation model is shown in Fig. 2, and the characteristics of all system parameters are explained in Table 1 and details of such parameters are shown in Table A.1. 


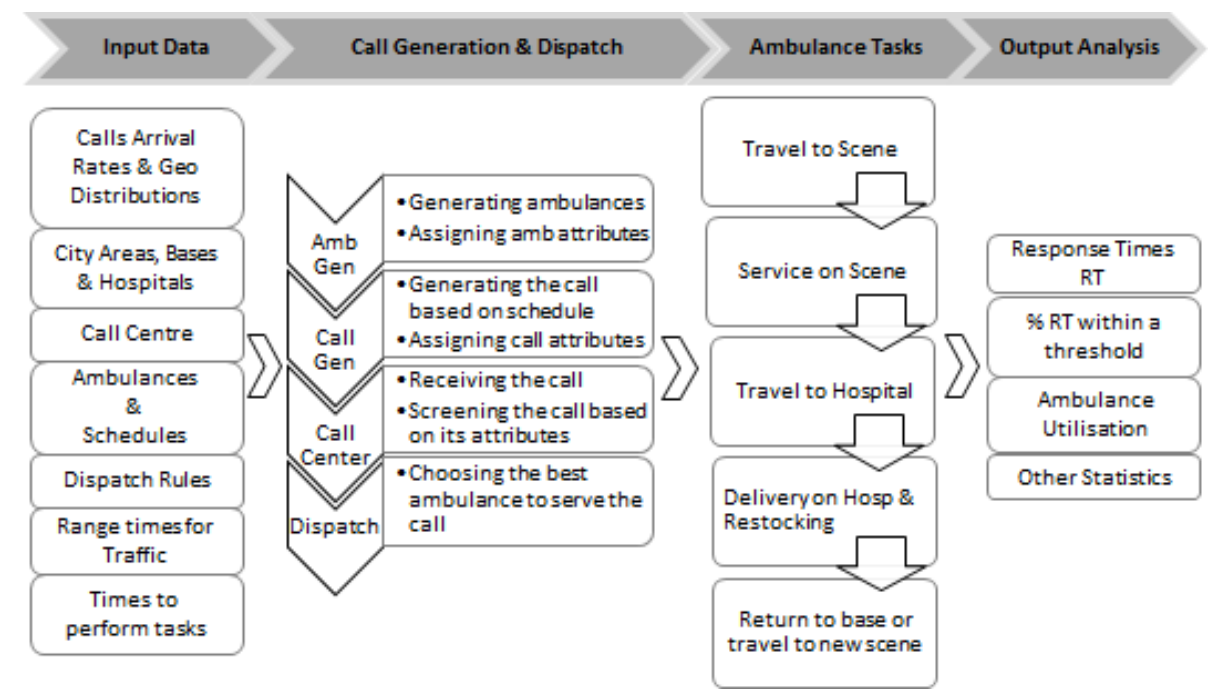

Figure 2: Description of the structure of the model

Ambulances and calls are the main entities of the system. Ambulances are permanent entities whilst calls are temporary.

Firstly, the inputs related to the characteristics of the system are read. Secondly, simulation starts and all ambulances are generated and attributes are assigned for each one. Thirdly, calls are generated based on schedule of call arrival and attributes are assigned to characterise each one. Operators receive calls and health technicians make triage based on the attributes of each one. Then emergency calls that require ambulance are routed to a dispatcher in order to choose the best ambulance to serve the emergency. After dispatch, ambulances perform tasks related to travel, service at the scene, delivery at hospital, and restocking. Ambulances may perform all these tasks or just some of them on each trip. Finally, after the simulation runs, the output analysis is processed in order to check the performance variables. The most important performance variables are: (i) general average response time; (ii) average response time for each category of call $\boldsymbol{c}$; (iii) percentage of response time less than a threshold for each category of call $\boldsymbol{c}$; and (iv) utilisation of ambulances of each type $\boldsymbol{u}$.

In this section, we focus on input data because a minimum level of standardisation is required in order to allow the reuse of the models.

\subsection{Call features}

The calls have several random and seasonal features related to arrival rates, geographical distribution, nature of health problem, level of threat to life, and the type of ambulance required. Calls can pass through three stages. First, it is necessary to eliminate the non-emergency and non-medical calls. Secondly, the emergency calls 
are classified take in account the nature of health problem, category of emergency, the ambulance needs, and types of units required. Finally, if dispatch is required, one ambulance is chosen based on the judgement of incident. Operators, technicians, and dispatchers in call centre are in charge of all these triage tasks.

Table 1

Input parameters

\begin{tabular}{|c|c|c|}
\hline Group & Parameter & Description \\
\hline \multirow[t]{9}{*}{ Calls } & $\lambda_{t}$ & Arrival rate of calls for each hour $t$ of the week (calls/h) \\
\hline & $G_{r a}$ & Fraction of calls for area $a$ during time range $r$ \\
\hline & $N C$ & Fraction of non-emergency calls (non-medical and/or hoax calls) \\
\hline & $A C$ & Fraction of emergency calls that require an ambulance dispatch \\
\hline & $M C$ & Fraction of calls that cause missing dispatches \\
\hline & $H C$ & Fraction of calls that require delivery patients at hospitals \\
\hline & $L C_{c}$ & Fraction of emergency calls for each category $\boldsymbol{c}$ (life-threatening classes) \\
\hline & $E C_{e}$ & Fraction of emergency calls for each nature $\boldsymbol{e}$ (nature of emergency) \\
\hline & $U C_{u}$ & Fraction of emergency calls that require ambulance of type $u$ \\
\hline RRV requirement & $R R_{c}$ & Fraction of each category $c$ that requires an RRV \\
\hline Traffic & $S T_{r}$ & Starting time of time range $\boldsymbol{r}(1 \leq \boldsymbol{r} \leq 4$ for weekdays and $5 \leq \boldsymbol{r} \leq 8$ for weekends) \\
\hline \multirow{6}{*}{$\begin{array}{l}\text { Ambulance and } \\
\text { Journey }\end{array}$} & $S_{r u}$ & Average ambulance speed for ambulance type $\boldsymbol{u}$ and time range $\boldsymbol{r}(\mathrm{km} / \mathrm{h})$ \\
\hline & $S F_{a}$ & Speed factor for area $\mathbf{a}$ \\
\hline & $J R_{u}$ & Number of trips between restocking for ambulance type $\boldsymbol{u}$ \\
\hline & $F$ & Distance conversion factor to correct Euclidian distance for the city \\
\hline & $Q_{u}$ & Total number of ambulances of type $\boldsymbol{u}$ \\
\hline & $N A_{\text {but }}$ & Number of ambulance of type $\boldsymbol{u}$ in each base $\boldsymbol{b}$ during the hour $\boldsymbol{t}$ of the week \\
\hline \multirow{5}{*}{$\begin{array}{l}\text { Personnel, Areas, } \\
\text { Bases and } \\
\text { Hospitals }\end{array}$} & $N R_{f}$ & Number of resource $\boldsymbol{f}$ at call centre (operators, technicians and dispatchers) \\
\hline & $X Y_{a p}$ & Coordinates $\boldsymbol{p}$ (lower-left and upper-right corners) of each area $\boldsymbol{a}$ \\
\hline & $P B_{b k}$ & Value of the parameter $\boldsymbol{k}$ for base $\boldsymbol{b}$ \\
\hline & $P H_{h j}$ & Value of the parameter $\boldsymbol{j}$ for hospital $\boldsymbol{h}$ \\
\hline & $D_{b a}$ & Notify whether base $\boldsymbol{b}$ can dispatch ambulances to area $\boldsymbol{a}$ (Boolean) \\
\hline \multirow{6}{*}{$\begin{array}{l}\text { Time to perform } \\
\text { Tasks }\end{array}$} & $\mathrm{TO}_{c}$ & Expression for spent time by Operator (category c) (seconds) \\
\hline & $T T_{u}$ & Expression for spent time by Technician (ambulance of type $\boldsymbol{u}$ ) (seconds) \\
\hline & $T D_{u}$ & Expression for spent time by Dispatcher (ambulance of type $u$ ) (seconds) \\
\hline & $T S_{u}$ & Expression for spent time on scene (ambulance of type $\boldsymbol{u}$ ) (minutes) \\
\hline & $T H$ & Expression for spent time to delivery patients at hospitals (minutes) \\
\hline & $T R_{u}$ & $\begin{array}{l}\text { Expression for spent time to replenish first aid materials and products } \\
\text { (ambulance type } \boldsymbol{u} \text { ) (minutes) }\end{array}$ \\
\hline
\end{tabular}

\subsubsection{Arrival rates}

Demand for emergency medical services differs for each city along with cycles. The most common of which, reflects differences within hours and days of week. Many cities also have peak demands in winter [14] and on special days such as New Year's Day. The weekly cycle, at least, with hourly structure must be taken into account to capture this seasonality.

Many studies have been carried out to forecast arrival rates in EMS. Matteson et al. [39] proposed a method using an hourly structure taking into account the day-ofweek and week-of-year effects. That was successful tested in Toronto, Canada.

The assumption that incoming calls are a non-homogeneous Poisson process with an hourly structure is widely used. We assume this hypothesis using a day-of-week effect to improve the quality of the simulation. 


\subsubsection{Geographical distribution of calls}

Calls have a non-uniform distribution around the city. The arrival rate may vary and it is time-dependent. Furthermore, calls may be related with socioeconomic conditions of the population. Earnest et al. [40] explored the relationship between socioeconomic status and the spatial distribution of ambulance calls in Singapore. We propose to divide the entire area of the city covered by ambulance system in cells as shown in Fig. 3.

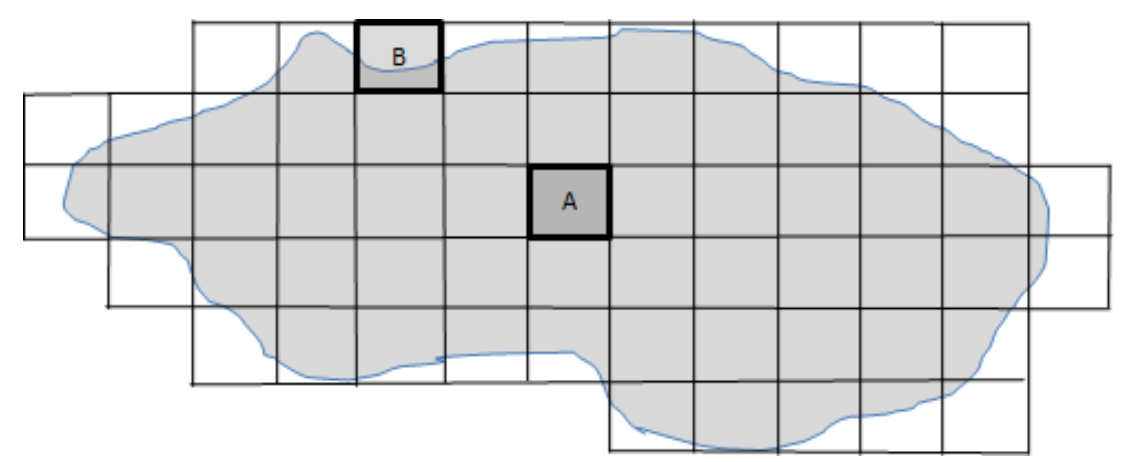

Figure 3: Area covered by the service

Each cell is identified by the coordinates of its lower-left corner and upper-right corner. We assume an empirical discrete distribution for distributing calls on cells. This distribution can vary according to time of day and day-of-week. When the area covers the entire rectangle (cell $A$ ) the coordinates of the call are assigned randomly inside the rectangle. Otherwise (cell B) the coordinates of the call are assigned randomly inside the partial area. Some systems, such those in the UK, use the postcode area instead of regular size areas.

\subsubsection{Types of emergency and ambulance requirements}

We have found distinct classes of emergency in the literature $[41,42,43]$ but we propose group them in four main classes related to nature of emergency and other four classes related to life-threatening. As some hospitals are not able to treat all kinds of emergency, the nature of emergency may be used to choose the hospital to deliver the patient. Classes related to life-threatening are used to prioritise the service.

On the other hand, each call requires a specific care unit, i.e., a specific type of ambulance and staff. We can group the ambulance and staff in classes, for example: (i) BLS: basic life support units, used to serve the simplest cases, (ii) ALS: advanced 
life support units, used to cover heart disease, cardiovascular / stroke and complex trauma and many others complex cases, (iii) MCS: mental care support units, used to serve complex psychiatric / drug cases, and (iv) RRV: rapid response vehicle used to make the first aids whist the patient wait for another type of ambulance.

\subsubsection{Other important attributes}

All these attributes are Booleans and the probabilities of occurrence are empirical. These attributes notify: i) whether the emergency requires an ambulance (some incidents do not require ambulance dispatch); ii) whether the call is not an emergency (hoax calls or non-emergency calls); iii) whether the incident requires the delivery of patient at hospital; and iv) whether the call will cause a missing dispatch, i.e., the care on scene is no longer necessary and the ambulance will make a missing travel.

\subsubsection{Calls generator}

The model generates entities calls based on arrival rate $\lambda_{t}$ and parameters shown in Table 1, are used to assign attributes to distinguish each one.

The first attributes, based on $G_{r a}$ and $X Y_{a p}$, are coordinates of the call and its type (based on $\mathrm{NC}$ ). The spent time by operators $\left(\mathrm{TO}_{c}\right)$ may depend of type of emergency. All non-emergency calls are disposed and emergency calls will receive an attribute to inform the dispatch needs (based on $A C$ ). Technicians spent some time $\left(T T_{u}\right)$ to triage calls and dispose those that do not require dispatch. All others calls will receive attributes, based on parameters in brackets, concerning the nature of emergency $\left(E C_{e}\right)$, category of the call $\left(L C_{c}\right)$, type of ambulance required $\left(U C_{u}\right), \mathrm{RRV}$ requirement $\left(R R_{c}\right)$, delivery requirement $(H C)$, and an attribute used to decide if the dispatch will be missed or not $(M C)$. After the screening process, the call is routed to dispatcher, who spent some time $\left(T D_{u}\right)$ to choose the unit. The number of operators, technicians, and dispatchers are given by $N R_{f}$.

\subsection{Ambulance features}

The entities ambulances are generated using $Q_{u}$ and the model uses a calendar schedule, based on $N A_{b u t}$, to allocate ambulances at bases around the city.

\subsubsection{Ambulance dispatch}


In static dispatch, the ambulance will go to scene from the nearest base, which has the requested type of ambulance. After the service on scene, the ambulance goes back to the base and stay there waiting for the next dispatch. Osborne et al. [44] analysed the real needs for advanced life support in seizure occurrences and the impact of these allocations on the system's performance. In multi-location dispatch, the allocation to new service can take place as soon after the ambulance finishing the current service, i.e., the ambulances become available just after they deliver the patient at hospital or after finishing the service on scene. In this approach, ambulances do not have to return to their bases before the next dispatch.

In dynamic dispatch, ambulances may be rerouted after the dispatch to serve a more critical call, i.e., the ambulances remain available even after dispatched. Billhardt et al. [45] proposed a strategy that combines a dynamic redeployment and allocation of ambulances in order to improve the performance of an EMS in the region of Madrid. Lim et al. [46] made a review of dynamic dispatch policies and they compared the advantages and disadvantages of each type. Dynamic dispatch requires fewer ambulances because it is more efficient but if the actual number of trips rerouted is not significant, the performance is not much better than multi-location dispatch. If rerouting does not occur frequently, we can use a multi-location dispatch as a good approximation for dynamic dispatch. Advantages of this approach are simple modelling and no GIS tools requirement.

In our model, we use a multi-location dispatch. The dispatch system maintains a list of every available ambulance and it determines the estimated time for each one to arrive on scene. The dispatched ambulance should belong to a base that allows dispatching to the area of the incident (parameter $D_{b a}$ ).

For each ambulance in the list, there is an associated release time and an associated release location. The ambulance may be released from a prior scene, from hospital or from base depending of the current time and characteristics of prior served emergency. If the ambulance is on the base, the release time is the current simulation time; otherwise, the release time will be greater than current simulation time. The system calculates the Euclidian distance between the releasing point of each ambulance and point of emergency site. We use a factor $F$ to correct the Euclidian distance and estimate the real distance. This factor take in account the topography and street design of the city and it is estimated using a GIS [1]. The average speed, used to estimate the travel time, can be time-dependent and different 
for each type of unit and each area of the city $\left(S_{r u}\right)$. Then, we should correct it using a speed factor $\left(S F_{a}\right)$.

When an emergency requires an ambulance RRV (usually category A - immediately life-threatening), the system search for a RRV and a regular ambulance for the same emergency. Both ambulances are sent to the site, but RRV probably will arrive sooner and it will wait on scene until the arrival of second ambulance. Thereafter, $\mathrm{RRV}$ returns to its base or to another scene.

The system chooses the nearest hospital that supplies the required care for the emergency (based on $P H_{h j}$ ). The system also estimates the travel time between the scene and the hospital and between the hospital and the base. Such times are used to estimate the next release time of the ambulance.

\subsubsection{Ambulance journey}

A regular journey has three sections: i) from the base to the scene, ii) from the scene to the hospital and iii) from the hospital to the base. Hospitals have a pre-defined capacity of beds to receive each type of emergency. If the hospital has no beds available, the ambulance will be diverted to other hospital. Ambulance diversion - AD causes delays in the system and must be avoided. AD has impact in transport times [47] and strategies to minimise its effects have been studied using simulation [48, 49, $50,51,52]$. Usually models of ambulance services do not capture this phenomenon because it is more pertinent for models of Emergency Departments that aim analysing the waiting time for admission in hospitals.

\subsubsection{Traffic}

The car traffic follows typical weekly seasonality patterns and it influences the speed of ambulances. It is not necessary an hourly structure neither day-of-week effect to capture such seasonality. We divide the week into two types of days: weekdays and weekends. The time ranges for car traffic should take on account night-time hours, morning peak hours, daytime hours and evening peak hours. Obviously the starting time $\left(S T_{r}\right)$ of each range depends on habits, culture and transportation infrastructure of the city. Fig. 4 shows an example of car traffic seasonality. 


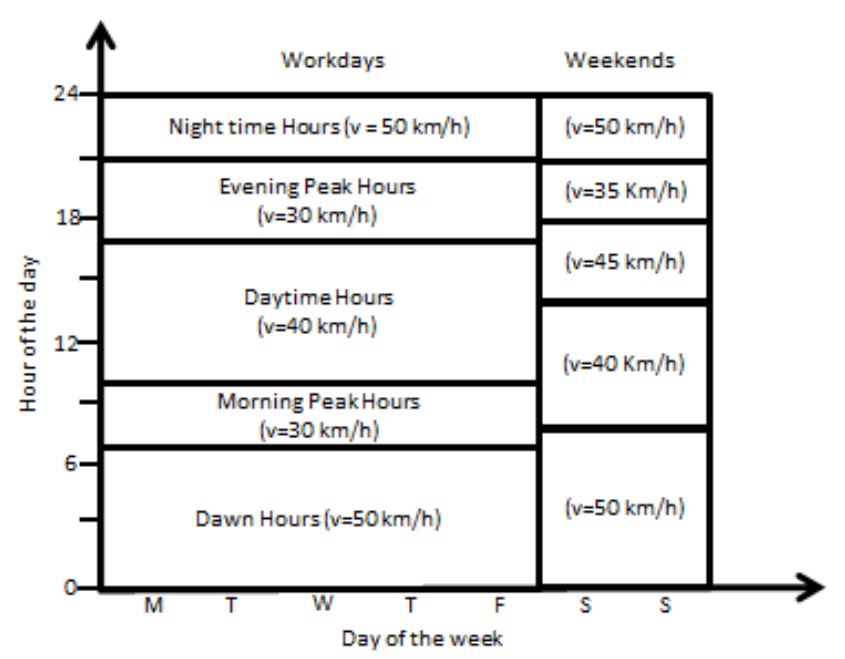

Figure 4: Example of traffic seasonality

\subsubsection{Service on scene, delivery at hospital and restocking}

The service is processed (duration $T S_{u}$ ) when ambulance arrives on scene. After the service on scene, the most likely destination is the hospital in order to deliver the patient (duration $T H$ ), but the ambulance may go back to its base or may be dispatched to serve a new event.

First aid materials and other products used in the ambulance should be replenished (duration $T R_{u}$ ) after a certain number of journeys $\left(J R_{u}\right)$.

\subsection{Data Collection}

The most important and most used performance measure for EMS is the average response time $-R T$ [20]. The response time is widely used because this performance measure is usually associated with patient survival $[53,54,55,56,57,58]$.

From a manager's point of view, the objective is operating the system with minimum cost and providing services of good quality, e.g., a high percentage of response times less than a threshold. Managers closely monitor all performance measures, such as utilisation of ambulances and utilisation of resource of call centres.

\subsection{Optimisation for simulation}

Managers have to choose the lowest cost configuration of the system to provide high quality services. They have to decide where locate the bases, the allocation of ambulances (calendar schedules), and number of personnel involved in operations of call centre. Many times they have to choose the best configuration using scarce resources. This decision is quite complex because the problem is combinatorial. 
In order to the simulation model estimate the best configurations for the system, we have to implement a simulation-optimisation strategy. We can use a classical strategy [38] to evaluate the best configuration. The schematic design of this strategy can be seen in Fig. 5 .

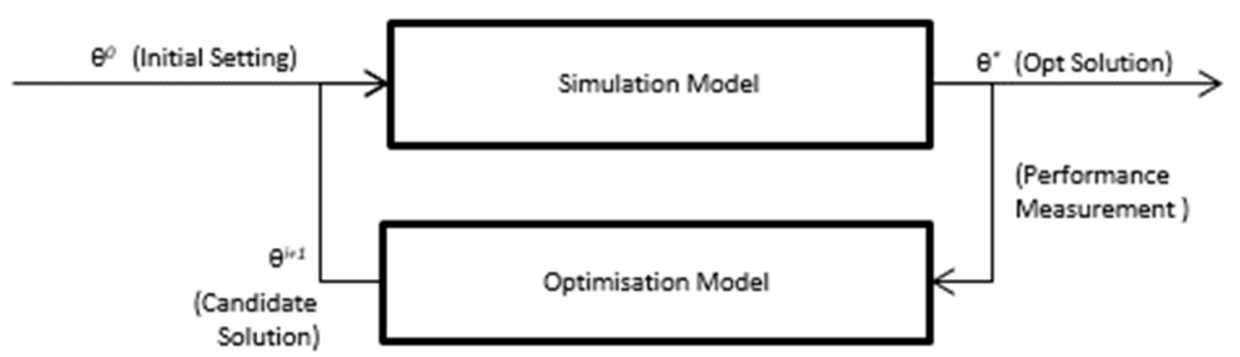

Figure 5: Optimisation for simulation scheme

The optimisation model cannot be written in the standard way, i.e., as a deterministic optimisation model, because some constraints are implicitly embedded into the simulation model and the evaluation of objective value and/or constraints can only be obtained by simulation. The candidate solution shown in Fig. 5 is obtained by heuristics, i.e., it has not been optimised in a formal sense.

The objective function given by (1), is to find the "best" (quotes is to denote nonformal sense) configuration for the system.

$$
\operatorname{Min}_{\theta \in \Theta} K(\theta)=E[K(\theta, \omega)]
$$

where $\theta$ is the set of input variables (candidate solution); $\omega$ is the replication; $k(\theta, \omega)$ is the sample performance measurement; $E[\kappa(\theta, \omega)]$ is the expected value of $k(\theta, \omega)$. The set of input variables is known as controls and performance measurements are known as responses. The objective of optimisation for simulation is searching for the best set of inputs $\theta$ that optimises the performance of the system under specific conditions (constraints). This objective often involves costs or performance of the system. Let $P B_{b k}$ be the set of characteristics of ambulance bases. $P B_{b 1}$ is a Boolean to inform if the base $b$ will be activated. $P B_{b 2}$ and $P B_{b 3}$ are coordinates of base $b$. Let $Q_{u}$ be the total number of ambulance type $u$. Let $\beta_{b}$ be the costs of activation of each base $b$, let $\eta_{u}$ be the cost of ambulance type $u$, and let $\varphi_{f}$ be the costs of resources $f$ at call centre. 
If we have many possible locations to set bases on the city and we wish the best configuration for the system, we can use the optimisation for simulation model given by (2) to (13).

$$
\operatorname{Min} Z=\sum_{b=1}^{n_{b}} \beta_{b} P B_{b 1}+\sum_{u=1}^{4}\left(\eta_{u} \cdot Q_{u}\right)+\sum_{f=1}^{3}\left(\varphi_{f} \cdot N R_{f}\right)
$$

Subject to:

$$
\begin{aligned}
& Q_{u}-\sum_{b=1}^{n_{b}} N A_{b u t} \geq 0 \quad \forall u, \forall t \\
& R T_{c} \leq R T_{c}^{*} \quad \forall c \\
& U T_{u} \leq U T_{u}^{*} \quad \forall u \\
& P T_{c} \geq P T_{c}^{*} \quad \forall c \\
& N R_{f} \geq N R_{f}^{-} \quad \forall f \\
& N A_{b u t} \geq N A_{b u t}^{-} \quad \forall b, \forall u, \forall t \\
& Q_{u} \geq Q_{u}^{-} \quad \forall u \\
& N R_{f} \leq N R_{f}^{+} \quad \forall f \\
& N A_{b u t} \leq N A_{b u t}^{+} \quad \forall b, \forall u, \forall t \\
& Q_{u} \leq Q_{u}^{+} \quad \forall u \\
& P B_{b 1} \text { is Boolean } \quad \forall b
\end{aligned}
$$

In this model, $P B_{b 1}, Q_{u}, N R_{f}$, and $N A_{b u t}$, are the controls and $R T_{c}, P T_{c}$, and $U T_{u}$ are the responses. Constraint (3) denotes the relationship between $Q_{u}$ and $N A_{b u t}$ (the maximum number of available ambulances type $\boldsymbol{u}$ in each hour-of-week should be $Q_{u}$ ). Constraints (4) and (5) set upper bounds to response time $R T_{c}$ for each category $c$ and utilisation of ambulances $U T_{u}$. Constraint (6) set lower bound to percentage of response time $P T_{c}$ less than a threshold for each category $c$. Constraints $(7)$ to (12) set lower and upper bounds to controls. Constraint (13) denotes $P B_{b 1}$ is Boolean variable.

As we can see, this is not an optimisation model in formal sense. There are no explicit analytical relationship between some controls and responses because the evaluation of responses is possible only by simulation and there are implicit dependence between such responses and controls. The simulation-optimisation loop works as follows: the results of each simulation are the input data for optimisation model, which solution is a new set of input for simulation model, which estimate new results and so on. The loop stops when pre-established condition is reached. We can use a commercial framework to implement this loop or we can developed a specific heuristics for this purpose. 
Obviously, the model shown here is only an illustration and we can write others models depending of our actual objective and constraints.

\section{Validation of the proposed method}

We developed a simulation model in Arena using the propositions explained in section 3 and the model was validated using real data from Belo Horizonte, a city located in Minas Gerais state, Brazil. We use MS Excel as interface between the simulation model and the users. All input and output data are read or written in a spreadsheet in order to ease the analysis. We use the blocks from ARENA templates rather than SIMAN language, but we had to use some Visual Basic code to implement the dispatch rules. The data, extracted from Silva [1], are from October/2006 until August/2009. The population of the city is around 2,500,000 inhabitants and the area covered by the system is $331 \mathrm{Km}^{2}$. The difference between the model proposed here and the model developed by Silva [1] is that his model is not generic and it was built specifically to analyse some aspects of EMS of Belo Horizonte. The city has nine administrative regions and the ambulance service used, on that occasion, 21 hospitals and emergency care units and 23 ambulances, which are spread around the bases in the city. The system does not use a calendar schedule to change the allocation of ambulances throughout the day or week. Table A.2 show the number of ambulances ALS, BLS and MCS in each base.

\subsection{Call features}

In this case, time ranges for weekdays and weekends are the same. The geographical distribution of calls in each region is shown in Fig. 6 . The hourly arrival rate of the calls on each day-of-week is shown in Fig. 7.

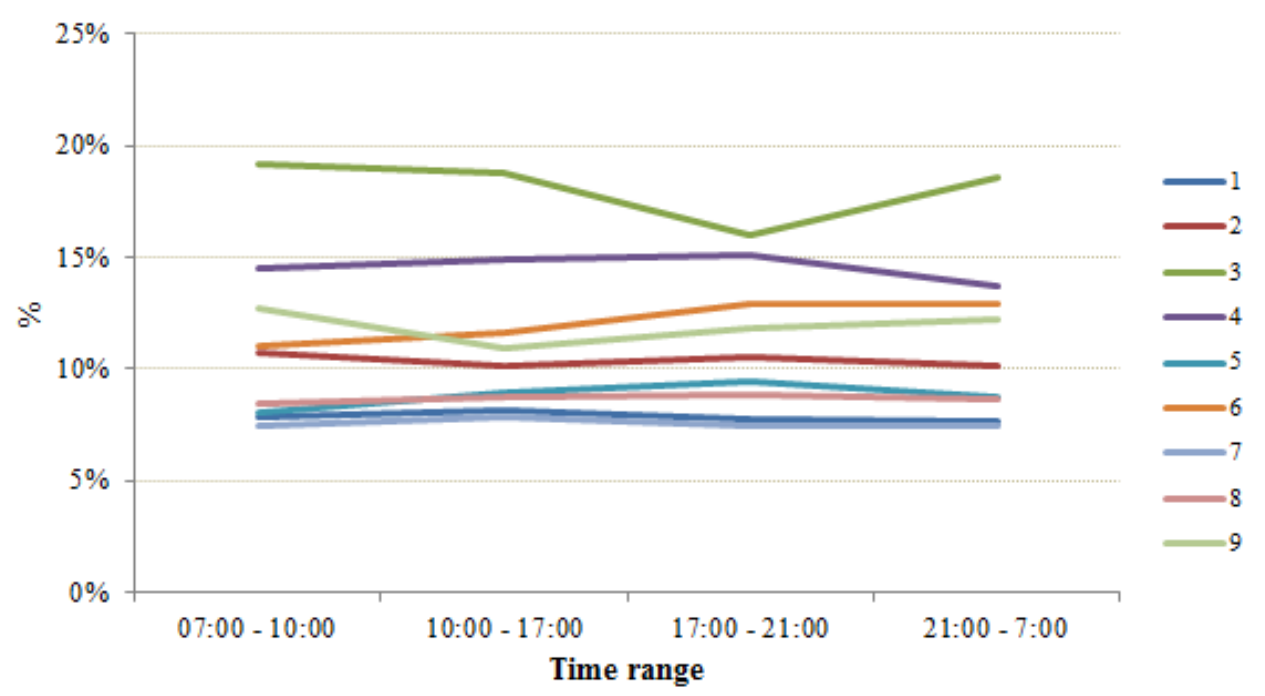


Figure 6: Distribution of calls for regions 1 to 9

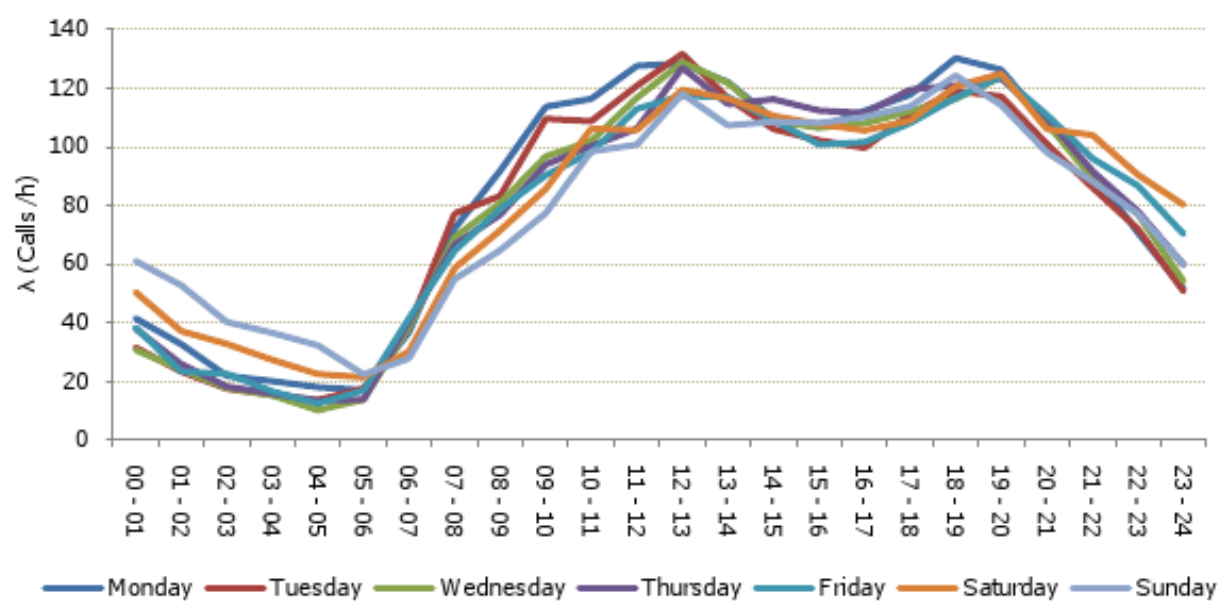

Figure 7: Arrival rates $-\lambda($ calls $/ \mathrm{h})$

The system receives more than 600,000 calls per year, including emergency calls and non-emergency calls. About $31.93 \%$ of calls are asking for ambulances, of which $40.84 \%$ resulting in an ambulance dispatch. About $90 \%$ of ambulance services deliver a patient at hospital, but in $5.21 \%$ of cases, the patient no longer requires the ambulance when it arrives.

The nature of emergency is split in four types and such classification is used by the system to choose the appropriate hospital for the emergency. The database of this EMS does not maintain registers regarding life-threatening, but emergencies requiring ALS have higher priority.

The computational implementation of the arrival of calls used an arrival schedule in order to contemplate the seasonality shown on Fig. 7. We modelled the geographical distribution of calls using the empirical distribution obtained through the data shown in Fig. 6. All others characteristics of calls are modelled using empirical distributions based on historical data.

\subsection{Ambulance features}

The average speed for each region is shown in Table A.3, estimated using Google Maps. We can see that the city has heavy car traffic and speed is not high. These estimations are for regular cars, not for ambulances. We use these speeds only for peak hours on weekdays. Ambulances travel faster than cars because they do not stop on red lights and the other vehicles give the way. Then, for other time ranges, 
we use speeds $10 \%$ to $30 \%$ faster. The ambulance system of this city does not use RRVs.

The first aid materials in ambulance should be replenished after a certain number of journeys as we can see in Table A.4. In the model, we consider ambulances always restock at a hospital just after finishing their journey.

\subsection{Call centre}

The human resources of the operational centre are six call operators, five physicians and three dispatchers. Firstly, operators receive the calls and they forward only the medical calls $(31.97 \%)$ to the physicians, who analyse the emergency and decide if an ambulance is necessary. If physicians decide to dispatch an ambulance, they also decide the type of ambulance should supply the event and forward their decision to dispatchers, who choose the best ambulance. Table A.4 shows the percentage of each type of ambulance is dispatched. All times required to execute tasks inside and outside call centres can be found in Silva [1].

\subsection{Dispatch rules and ambulance features}

The dispatching is under multi-location rules. The model estimates the time required to each ambulance arrive on scene using Euclidian distance corrected by factor $F$, estimated using Google Maps and average speed for the region.

The hospital in which the patient will be delivered is the nearest and it must provide the care required by the nature of emergency.

We supposed that maintenance of ambulances occurs on the same way proposed by Silva [1], i.e., average of three maintenances per day for all ambulance fleet.

The complex rules of dispatch make its implementation almost impossible if we use exclusively the ordinary blocks of ARENA. To implement the dispatch system we use a special block that allow us to include Visual Basic code inside it.

\section{Results and discussion}

\subsection{Results for current system}

After we run some pilot projects, we decide to run a simulation using a warm-up of 15 days and ten replications of 30 days. Fig. 8 shows that the system reaches the steady state even before 15 days (360 hours). We used response time and utilisation of ambulances (BLS and ALS) to evaluate the performance of the system. We did not 
use the utilisation of mental care units because the number of emergencies supplied by this unit is insignificant and the utilisation is very low.

Table 2 shows the values of response time (minutes); utilisation of BLS units (\%) and utilisation of ALS units (\%) for each replication. The average values (Avg), standard deviation (SD), and confidence interval (CI) are shown as well.

The response time found by Silva [1] for a similar scenario is equivalent. The response time obtained by our model is only $2.06 \%$ greater than the actual average response time, which is $19.92 \mathrm{~min}$ [1]. Utilization of ambulances are slightly greater than the results of Silva [1].

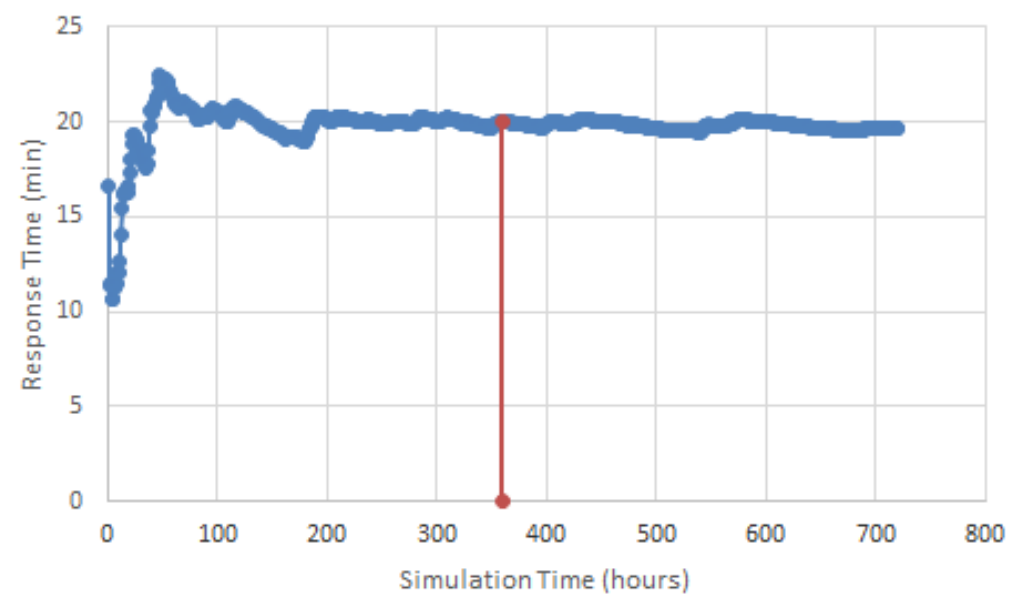

Figure 8: Warm-up period

As we can see, the response time in Belo Horizonte in 2009 was very high compared to international standards. The model does not estimate the response time by category of call (life-threatening) because we have no information about category of call in the database of the system. There is a low percentage of $R T$ within 15 minutes $(41.96 \%)$ for all categories of calls. This fact occurs due to the delay until dispatching and very large travel times. The tasks in call centre spend too much time and travel is time-consuming due to network design of streets and heavy traffic.

Thus, to reduce the response time it is necessary to increase the number of ambulances and spread them around several bases in the city, or to adopt a schedule to change the base of ambulances during the hour-of-day and day-of-week in order to improve the ambulance logistics. Furthermore, the tasks in call centre should be changed in order to reduce the elapsed time between the arrival of call and the dispatch of an ambulance. 


\begin{tabular}{|c|c|c|c|c|c|c|}
\hline \multirow[b]{2}{*}{ Replication } & \multicolumn{2}{|c|}{ Proposed Model } & \multicolumn{4}{|c|}{ Silva Model (2010) } \\
\hline & RT(min) & UB (\%) & UA (\%) & RT(min) & UB (\%) & UA (\%) \\
\hline 1 & 20.53 & 58.19 & 13.44 & 19.40 & 56.33 & 10.39 \\
\hline 2 & 20.04 & 56.73 & 12.41 & 21.12 & 54.48 & 11.84 \\
\hline 3 & 20.04 & 56.38 & 11.73 & 21.93 & 56.53 & 10.84 \\
\hline 4 & 20.16 & 57.31 & 13.28 & 19.95 & 53.24 & 12.98 \\
\hline 5 & 22.11 & 58.23 & 11.79 & 20.38 & 58.95 & 13.14 \\
\hline 6 & 20.52 & 57.30 & 13.17 & 21.44 & 54.12 & 12.09 \\
\hline 7 & 20.88 & 57.97 & 12.38 & 20.57 & 55.59 & 10.77 \\
\hline 8 & 19.67 & 56.53 & 12.74 & 19.11 & 54.28 & 11.61 \\
\hline 9 & 19.59 & 56.98 & 11.57 & 20.07 & 54.87 & 12.64 \\
\hline 10 & 19.79 & 56.96 & 13.36 & 20.18 & 54.79 & 11.24 \\
\hline Avg (min) & 20.33 & 57.26 & 12.59 & 20.42 & 55.32 & 11.75 \\
\hline SD (min) & 0.75 & 0.67 & 0.72 & 0.88 & 1.63 & 0.96 \\
\hline $\mathrm{Cl}(\alpha=0.05)$ & \pm 0.53 & \pm 0.48 & \pm 0.51 & \pm 0.63 & \pm 1.16 & \pm 0.69 \\
\hline
\end{tabular}

In London, for example, the current target for category A (immediately threateninglife) calls is $75 \%$ of response times must be within 8 minutes and $95 \%$ of response times must be within 19 minutes. Between April 2011 and March 2012, the London Ambulance Service reached $75.74 \%$ of category A calls within 8 minutes. The UK system uses RRVs and the number of ambulances in each base may vary throughout the day and week.

The Brazilian government and the City of Belo Horizonte have worked together in order to improve the ambulance service. New ambulances units were acquired since 2010 and the ambulance system and fire brigade system have been integrated in order to improve the quality of service. Unfortunately, we have no data to analyse the system after those changes.

\subsection{Results using rules applied in UK system}

One important feature of a generic modelling is the ability to expand the model by interfacing it to another. As the proposed model is quite generic, it is possible to investigate the effects of the adoption of some rules applied in ambulance systems of UK in the system of Belo Horizonte. We try two rules by way of illustration. Firstly, we adopt a calendar schedule to define ambulance availability and secondly, we explore the use of RRV for emergencies that require advanced life support units (ALS). In the first experiment, we simulate the ambulance system of Belo Horizonte with no increase in the number of ambulances but we use a calendar schedule to define the number of ambulance in each base during each hour of the week. After an analysis based on demand, base locations and allocations of ambulances, we decide to change the static allocation of ambulance shown in Table A.4 for a scenario using the following calendar schedule: 
- Removing 1 BLS from base 14 and allocate it to base 4 between 11:00h and

13:00h and between 17:00h and 19:00h all days of week;

- Removing 1 BLS from base 7 and allocate it to base 4 between 00:00h and 07:00h and on base 5 between 07:00h and 10:00h all days of week;

- Removing 1 BLS from base 6 and allocate it to base 5 between 07:00h and 10:00h all days of week;

- Removing 1 BLS from base 6 and allocate it to base 5 between 11:00h and 13:00h on Mondays and Tuesdays.

This calendar schedule increases the number of ambulances on bases 4 and 5 , mainly on peak hours and on weekdays with high demands. Those bases are located in the region that has the higher demand all the time (area 3). Then, this change is supposed to improve the performance of the system. The simulation corroborates this supposition and we find an average $R T$ reduced in 9 of 10 replications. The average $R T$ was reduced by $3.79 \%$ (46.2 seconds).

In the second experiment, we used the same calendar schedule and we suppose the use of 5 RRV's units working together 5 ALS units. ALS is supposed to supply the most complex cases and an RRV would work in order to reduce the average $R T$ for this type of unit (15.72 \pm 0.14 minutes). We supposed RRV moves $25 \%$ faster than a regular ambulance. In a similar way of UK, RRV units would be dispatch always an emergency requires an ALS and it should stay on scene until the arrival of ALS. The simulation results show an average $R T=13.25 \pm 0.03$ minutes, i.e., a decrease of $15.71 \%$ (2.47 minutes) on RT of ALS units. The general response time (for all types of ambulances) in this scenario was $18.97 \pm 0.33$ minutes, i.e., $6.69 \%$ less than the original scenario (20.33 \pm 0.53 minutes).

These preliminary results must be confirmed by further experiments but we can see some possibilities to improve the performance of the system making simple changes and some of them require no additional cost. Furthermore, this scenario analysis was useful to show how we can take advantage of generic models to expand the system capability.

\section{Conclusions}

In this work, we propose a method to build generic simulation models to analyse ambulance service system by simulation and we have applied it to compare two aspects of provision between Brazil and UK. We explain the most important input data and how we should handle them and the main guidelines to build simulation 
models regardless of the simulation framework being used. We explain the main performance measures under point of view of patients and managers. In addition, we proposed some guidelines to build optimisation for simulation models to check alternatives in order to get good configurations for the system. Furthermore, we built a simulation model using the proposed method and implement it using a commercial simulation framework. The model was validated using real data and results were quite satisfactory. The proposed model seems be comprehensive and easy to use. We take the advantage of reuse and standardisation to check the impact of rules from UK on Brazilian system in order to investigate likely improvements.

We believe that the guidelines proposed in this paper will be useful for building simulation models more quickly so that the models can be easily reused with minor adjustment. In doing this, we have demonstrated model reuse in two regions which use very different systems and policies. To the best of our knowledge, this is the first time that this has been done.

\section{Acknowledgments}

The authors thank Paul Harper from Cardiff University, Khairy Kobbacy from Salford University and their respective staffs, and Julie Eatock from Brunel University London, for their comments and suggestions. The authors acknowledge support of this work through the MATCH Programme (EPSRC Grants GR/S29874/01 EP/F063822/1 \& EP/G012393/1), although the views expressed are entirely theirs. L. R. Pinto acknowledge support through the CAPES Foundation (Fellowship Grant BEX-0870/11-0) and FAPEMIG Foundation (Grant TEC - APQ-02219-12).

\section{References}

[1] P. M. S. Silva, Análise do Serviço de Atendimento Móvel de Urgência (SAMU) via simulação e otimização (Master's Thesis). Universidade Federal de Minas Gerais. Belo Horizonte, Brazil, 2010 (in portuguese).

[2] P. M. S. Silva, L. R. Pinto, Emergency Medical System Analysis by Simulation and Optimization, in: Johansson, B., Jain, S., Montoya-Torres, J., Hugan, J., Yücesan, E. (Eds.), Proceedings of the 2010 Winter Simulation Conference (2010), p. $2422-2432$.

[3] M. Singer, P. Donoso, Assessing an ambulance service with queuing theory. Computers \& Operations Research 35 (2008), 2549 - 2560. 
[4] A. P. Iannoni, R. Morabito, A multiple dispatch and partial backup hypercube queuing model to analyze emergency medical systems on highways.

Transportation Research Part E 43 (2007), 755 - 771.

[5] R. A. Takeda, J. A. Widmer, R. Morabito, Analysis of ambulance decentralization in an urban emergency medical service using the hypercube queueing model.

Computers \& Operations Research 34 (2007), 727 - 741.

[6] R. Morabito, F. Chiyoshi, R. D. Galvão, Non-homogeneous servers in emergency medical systems: Practical applications using the hypercube queueing model. Socio-Economic Planning Sciences 42 (2008), 255 - 270.

[7] A. P. Iannoni, R. Morabito, C. Saydam, An optimization approach for ambulance location and the districting of the response segments on highways. European Journal of Operational Research 195 (2009), 528 - 542.

[8] E. Almehdawe, B. Jewkes, Q-M. He, A Markovian queueing model for ambulance offload delays. European Journal of Operational Research 226 (2013), 602-614.

[9] H. Morohosi, T. Furuta, Hypercube simulation analysis for a large-scale ambulance service system, in: C. Laroque, J. Himmelspach, R. Pasupathy, O. Rose, and A.M. Uhrmacher. (Eds.), Proceedings of the 2012 Winter Simulation Conference (2012).

[10] V. Schmid, K. F. Doerner, Ambulance location and relocation problems with time-dependent travel times. European Journal of Operational Research 207 (2010), 1293 - 1303.

[11] M. Maleki, N. Majlesinasab, M. M. Sepehri, Two new models for redeployment of ambulances. Computers and Industrial Engineering (2014) http://dx.doi.org/10.1016/j.cie.2014.05.019 (in press).

[12] J. Naoum-Sawaya, S. Elhedhli, A stochastic optimization model for real-time ambulance redeployment. Computers \& Operations Research 40 (2013), 19721978.

[13] M. T. Maxwell, M. Restrepo, S. G. Henderson, H. Topaloglu, Approximate Dynamic Programming for Ambulance Redeployment. INFORMS Journal on Computing 22 (2) (2010), $266-281$.

[14] R. Berchi, G. Fontana, A. Pagliosa, R. Bonora, G. Sesana, A five steps methodology for an Ambulance Planning, in: Proceedings of the 2010 IEEE Workshop on Health Care Management (2010), p. 1 - 5. 
[15] H. Morohosi, T. Furuta, Optimization model and simulation for improving ambulance service system. 11th International Symposium on Operations Research and its Applications in Engineering, Technology and Management (2013), 23-25.

[16] C. E. Bell, D. Allen, Optimal Planning of an Emergency Ambulance Service. Socio-Economic Planning Sciences 3 (1969), 95 - 101.

[17] E. S. Savas, Simulation and Cost-effectiveness Analysis of a New York's Emergency Ambulance Service. Management Science 15 (12) (1969), B608 B627.

[18] J. A. Fitzsimmons, An emergency medical system simulation model, in: Proceedings of the 1971 Winter Simulation Conference Proceedings (1971), p. $18-25$.

[19] C. Swoveland, D. Uyeno, I. Vertinsky, R. Vickson, A simulation-based methodology for optimization of ambulance service policies. Socio-Economic Planning Sciences 7 (1973), 697 - 703.

[20] J. A. Fitzsimmons, R. S. Sullivan, Establishing the level of service for public emergency ambulance systems. Socio-Economic Planning Sciences 13 (1979), $235-329$.

[21] O. Fujiwara, T. Makjamroen, K. K. Gupta, Ambulance deployment analysis: a case study of Bangkok. European Journal of Operational Research 31 (1987), 9 $-18$.

[22] S. G. Henderson, A. J. Mason, Estimating Ambulance Requirements in Auckland, New Zeland, in: Farrington, P. A., Nembhard, H. B., Sturrock, D. T., Evans, G. W. (Eds.), Proceedings of the 1999 Winter Simulation Conference (1999), p. $1670-1674$.

[23] O. Kock, H. Weigl. Modeling Ambulance Service of the Austrian Red Cross, in: Chick, S., Sánchez, P. J., Ferrin, D., Morrice, D. J. (Eds.) Proceedings of the 2003 Winter Simulation Conference (2003), pp. 1701 - 1706.

[24] S. Su, C. L. Shih, Modeling an Emergency Medical Services System using Computer Simulation. International Journal of Medical Informatics 72 (2003), 57 -72 .

[25] C. H. Wu, K. P. Hwang, Using a Discrete-event Simulation to Balance Ambulance Availability and Demand in Static Deployment Systems. Academic Emergency Medicine 16 (12) (2009), 1359 - 1366. 
[26] L. Aboueljinane, E. Sahin, Z. Jemai, J. Marty, A simulation study to improve the performance of an emergency medical service: Application to the French Val-deMarne department. Simulation Modelling Practice and Theory 47 (2014), 46 - 59.

[27] L. Zhen, K. Wang, H. Hu, D. Chang, A simulation optimization framework for ambulance deployment and relocation problems. Computers and Industrial Engineering 72 (2014), 12 - 23.

[28] R. Aringhieri, An Integrated DE and AB simulation model for EMS management, in: Proceedings of the 2010 IEEE Workshop on Health Care Management (2010), p. $1-6$.

[29] ORGANIZACIÓN PANAMERICANA DE LA SALUD (OPAS), Información y Análisis de Salud: Situación de Salud en las Américas: Indicadores Básicos 2009. Washington, D.C., 2009 (in Spanish).

[30] UNITED NATIONS POPULATION FUND (UNFPA), State of World Population 2011: People and possibilities in a world of 7 billion. New York, 2011.

[31] S. Brailsford, P. Harper, B. Patel, M. Pitt, An analysis of the academic literature on simulation and modelling in health care. Journal of Simulation 3 (2009), $130-$ 140.

[32] M. Jahangirian, A. Naseer, L. Stergioulas, T. Young, T. Eldabi, S. Brailsford, B. Patel, P. Harper, Simulation in health-care: lessons for other sectors. Operational Research 12 (1) (2010), 45 - 55.

[33] J. Kujlis, R. J. Paul, L. K. Stergioulas, Can health care benefit from modeling and simulation methods in the same way as business and manufacturing has?, in: Henderson, S. G., Biller, B., Hsieh, M. -H, Shortle, J., Tew, J. D., Barton, R. R. (Eds.), Proceedings of the 2007 Winter Simulation Conference (2007), p. $1449-$ 1453.

[34] T. Young, J. Eatock, M. Jahagirian, A. Naseer, R. Lilford, Three critical challenges for modelling and simulation in healthcare. in: Rossetti, M. D., Hill, R. R., Johansson, B., Dunkin, A., Ingalls, R. G. (Eds.), Proceedings of the 2009 Winter Simulation Conference (2009), p. 1823-1830.

[35] M. M. Gunal, M. Pidd, Discrete event simulation for performance modelling in health care: a review of the literature. Journal of Simulation 4 (1) (2010), $42-51$.

[36] T. Eldabi, T. Young, Towards a framework for healthcare simulation, in: Henderson, S. G., Biller, B., Hsieh, M. -H, Shortle, J., Tew, J. D., Barton, R. R. (Eds.), Proceedings of the 2007 Winter Simulation Conference (2007), p. $1454-$ 1460. 
[37] D. Fone, S. Hollinghurst, M. Temple, A. Round, N. Lester, A. Weightman, K. Roberts, E. Coyle, G. Bevan, S. Palmer, Systematic review of the use and value of computer simulation modelling in population health and health care delivery. Journal of Public Health Medicine 25 (4) (2003), 325 - 335.

[38] M. C. Fu, Optimization for simulation: theory vs. practice. INFORMS Journal on Computing 14 (3) (2002), $192-215$.

[39] D. S. Matteson, M. W. McLean, D. B. Woodard, S. G. Henderson, Forecasting emergency medical service call arrival rates. The Annals of Applied Statistics 5 (2B) (2011), $1379-1406$.

[40] A. Earnest, S. B. Tang, N. Shahidah, M. E. H. Ong, Geographical variation in ambulance calls is associated with socioeconomic status. Academic Emergency Medicine 19 (2012), 180-188.

[41] A. B. Allen, B. G. Barnard, W. Falk, E. R. Higgs, J. G. McCracken, A study of waiting time in an emergency department. CMA Journal 109 (1973), 373 - 376.

[42] K. Siddharthan, W. J. Jones, J. A. Johnson, A priority queuing model to reduce waiting times in emergency care. International Journal of Health Care Quality Assurance 9 (5) (1996), 10 - 16.

[43] National Health System (NHS): London Ambulance Service, 2012, www.londonambulance.nhs.uk/about us/how we are doing/meeting our targets.as px, accessed on Aug. 04, 2014.

[44] A. Osborne, L. Taylor, M. Reuber, R. A. Grunewald, M. Parkinson, J. M. Dickson, Pre-hospital care after a seizure: evidence base and United Kingdom management guidelines. Seizure: Eur. J. Epilep. (2014). http://dx.doi.org/10.1016/j.seizure.2014.09.002 (in press).

[45] H. Billhardt, M. Lujak, V. Sánchez-Brunete, A. Fernández, S. Ossowski, Dynamic coordination of ambulances for emergency medical assistance services.

Knowledge Based Systems (2014).

http://dx.doi.org/10.1016/j.knosys.2014.07.006 (in press).

[46] C. S. Lim, R. Mamat, T. Braunl, Impact of ambulance dispatch policies on performance of emergency medical services. IEEE Transactions on Intelligent Transportation Systems 12 (2) (2011), 624 - 632.

[47] J. C. Pham, R. Patel, M. G. Millin, T. D. Kirsch, A. Chanmungan, The effects of ambulance diversion: A comprehensive review. Academic Emergency Medicine 13 (11) (2006), $1220-1227$. 
[48] A. R. Nafarrate, J. W. Fowler, T. Wu, Bi-Criteria Analysis on Ambulance Diversion Policies, in: Johansson, B., Jain, S., Montoya-Torres, J., Hugan, J., Yücesan, E. (Eds.), Proceedings of the 2010 Winter Simulation Conference (2010), p. $2315-2326$.

[49] A. R. Nafarrate, J. W. Fowler, T. Wu, Design of Centralized Ambulance Diversion Policies using Simulation-Optmization, in: Jain, S., Creasey, R. R., Himmelspach, J., White, J. P. (Eds.), Proceedings of the 2011 Winter Simulation Conference (2011), p. $1251-1262$.

[50] C-H Lin, C-Y Kao, C-Y Huang, Managing emergency department overcrowding via ambulance diversion: A discrete event simulation model. Journal of the Formosan Medical Association (2012), 1 - 8. http://dx.doi.org/10.1016/j.jfma.2012.09.007 (in press).

[51] A. Ramirez-Nafarrate, A. B. Hafizoglu, E. S. Gel, J. W. Fowler, Optimal control policies for ambulance diversion. European Journal of Operational Research 236 (2014), 298-312.

[52] A. Ramirez-Nafarrate, A. B. Hafizoglu, Comparison of ambulance diversion policies via simulation, in: C. Laroque, J. Himmelspach, R. Pasupathy, O. Rose, and A.M. Uhrmacher. (Eds.), Proceedings of the 2012 Winter Simulation Conference (2012).

[53] T. H. Blackwell, J. S. Kaufman, Response Time Effectiveness: Comparison of Response Time and Survival in an Urban Emergency Medical Services System. Academic Emergency Medicine 9 (4) (2002), 288 - 295.

[54] P. T. Pons, J. S. Haukoos, W. Bludworth, T. Cribley, K. A. Pons, V. J. Markovchick, Paramedic Response Time: Does It Affect Patient Survival? Academic Emergency Medicine 12 (7) (2005), 594 - 600.

[55] R. B. Vukmir, Survival from prehospital cardiac arrest is critically dependent upon response time. Resuscitation 69 (2006), 229 - 234.

[56] L. A. McLay, M. E. Mayorga, Evaluating emergency medical service performance measures. Health Care Management Sciences 13 (2010), 124 136.

[57] R. Sánchez-Mangas, A. García-Ferrrer, A. Juan, A. M. Arroyo, The probability of death in road traffic accidents. How important is a quick medical response? Accident Analysis and Prevention 42 (2010), 1048 - 1056. 
[58] C. O'Keeffe, J. Nicholl, J. Turner, S. Goodacre, Role of ambulance response times in the survival of patients with out-of-hospital cardiac arrest. Emerg Medical Journal 28 (2011), $703-706$. 


\section{APPENDIX}

\section{Table A.1}

Sets of input data

\begin{tabular}{|c|c|c|}
\hline Set & Range & Description \\
\hline $\boldsymbol{A}$ & $1,2 \ldots \mathrm{n}_{\mathrm{a}}$ & Area (cell) of the city \\
\hline$B$ & $1,2 \ldots n_{b}$ & Ambulance bases \\
\hline$H$ & $1,2 \ldots \mathrm{n}_{\mathrm{h}}$ & Hospitals \\
\hline $\boldsymbol{R}$ & $1,2, \ldots 8$ & $\begin{array}{l}\text { Time ranges: } 1 \text { to } 4 \text { for Weekdays and } 5 \text { to } 8 \text { for Weekends } \\
1 \text { (Niaht-times) } 2 \text { and } 6 \text { (Morning Peak) } 3 \text { and } 7 \text { (Davtime hours) } 4 \text { and } 8\end{array}$ \\
\hline$T$ & $1,2, \ldots 168$ & Hour-of-week ( $1=$ Sun $00: 00 \mathrm{~h}-$ Sun $01: 00 \mathrm{~h}$ to $168=$ Sat $23: 00 \mathrm{~h}-$ Sun $00: 00 \mathrm{~h})$ \\
\hline $\boldsymbol{U}$ & $1,2, \ldots 4$ & Type of ambulance ( $1=\mathrm{BLS}, 2=\mathrm{ALS}, 3=\mathrm{MCS}$ and $4=\mathrm{RRV})$ \\
\hline$E$ & $1,2, \ldots 4$ & Nature of emergency (medical class) \\
\hline $\boldsymbol{C}$ & $1,2, \ldots 4$ & Category of emergency (life-threatening level) \\
\hline $\boldsymbol{P}$ & $1,2 \ldots 4$ & Geographical coordinates of areas (lower-left and upper-right corner) \\
\hline$K$ & $1,2 \ldots 3$ & $\begin{array}{l}\text { Base settings: }(1=\text { Base status }(1=\text { Active, } 0=\text { Deactivate), } 2 \text { and } 3 \text { (Geographical coordinates } \\
\text { of the base }) \text { ) }\end{array}$ \\
\hline$J$ & $1,2 \ldots 7$ & $\begin{array}{l}\text { Hospital settings: }(1=\text { Hospital Status }(1=\text { Active, } 0=\text { Deactivate), } 2 \text { and } 3 \text { (Geographical } \\
\text { coordinates of the hospital), } 4 \text { to } 7 \text { (Availability for emergency types } 1 \text { to } 4 \text { respectively: } 1= \\
\text { Available, } 0=\text { Unavailable)) }\end{array}$ \\
\hline
\end{tabular}

Table A.2

Number of ambulances

\begin{tabular}{cccc}
\hline Base & BLS & ALS & MCS \\
\hline $\mathbf{1}$ & 0 & 1 & 0 \\
$\mathbf{2}$ & 1 & 1 & 0 \\
$\mathbf{3}$ & 1 & 1 & 0 \\
$\mathbf{4}$ & 0 & 1 & 0 \\
$\mathbf{5}$ & 0 & 1 & 0 \\
$\mathbf{6}$ & 1 & 0 & 0 \\
$\mathbf{7}$ & 1 & 0 & 0 \\
$\mathbf{8}$ & 1 & 0 & 0 \\
$\mathbf{9}$ & 1 & 0 & 0 \\
$\mathbf{1 0}$ & 1 & 0 & 0 \\
$\mathbf{1 1}$ & 2 & 0 & 0 \\
$\mathbf{1 2}$ & 1 & 0 & 0 \\
$\mathbf{1 3}$ & 1 & 0 & 0 \\
$\mathbf{1 4}$ & 1 & 0 & 0 \\
$\mathbf{1 5}$ & 1 & 0 & 0 \\
$\mathbf{1 6}$ & 1 & 0 & 0 \\
$\mathbf{1 7}$ & 1 & 0 & 0 \\
$\mathbf{1 8}$ & 1 & 0 & 0 \\
$\mathbf{1 9}$ & 1 & 0 & 0 \\
$\mathbf{2 0}$ & 0 & 0 & 1 \\
\hline Total & $\mathbf{1 7}$ & $\mathbf{5}$ & $\mathbf{1}$ \\
\hline
\end{tabular}

Table A.3

Average speed for each cell

\begin{tabular}{ccccccccccc}
\hline Region & $\mathbf{1}$ & $\mathbf{2}$ & $\mathbf{3}$ & $\mathbf{4}$ & $\mathbf{5}$ & $\mathbf{6}$ & $\mathbf{7}$ & $\mathbf{8}$ & $\mathbf{9}$ & Average \\
Speed (km/h) & 30.61 & 24.16 & 23.79 & 24.32 & 26.53 & 25.64 & 28.39 & 27.36 & 27.77 & 26.51 \\
\hline
\end{tabular}

Table A.4

Ambulance data

\begin{tabular}{ccc}
\hline Type of ambulance & \% Ambulance Dispatched & Number of trips between restocking \\
\hline BLS & 95.11 & 14 \\
ALS & 4.66 & 1 \\
MCS & 0.23 & 1 \\
\hline
\end{tabular}

